\title{
El debate de la medición digital: una piedra en el camino para la consolidación de la inversión publicitaria
}

\author{
Lidia MAEstro EsPínOlA ${ }^{1}$ \\ Universidad Internacional de La Rioja
}

Recibido: 06/11/15

Aceptado: 04/12/15

\begin{abstract}
Resumen
La llegada de ingresos provenientes de la distribución digital de la prensa continúa siendo reducida. A pesar de su volumen de lectores, el aumento de posibilidades dentro de los formatos publicitarios, la capacidad de medición del retorno de la inversión que ofrece el medio digital y la aparente unificación en la medición de audiencia que desde 2012 está presente en el mercado. De esta manera, los diferentes medios continúan sin conseguir rentabilizar la migración digital que cada día conlleva continuos gastos sin ofrecer beneficios. La audiencia como moneda de cambio en la comercialización de espacios publicitarios ha perdido todo su valor, pese al retorno de la inversión que ofrece. Este trabajo describe esta problemática y los cambios realizados antes y después del «fin del debate de la medición de audiencias» con el objetivo de poner en valor la venta de los espacios publicitarios digitales. En concreto se analiza el caso de los diarios digitales por su elevada lectura y sus escasos ingresos.
\end{abstract}

Palabras clave: mediciones, publicidad digital, diarios digitales, ingresos, compra programática.

\section{Digital advertising measuring under suspiction: a lack of standards}

\begin{abstract}
The incomes which arise out from the digital distribution of the press are still reduced. This situation is a fact despite the number of readers, the multiplication of possibilities within the advertising formats, the capacity of measuring the Return On Investments (ROI) on digital media and the unification of the criteria for measuring audiences since 2012. For these reasons, mass media cannot make digital migration profitable. Audience, as a bargain chip in the marketing of advertising space, has lost its value despite the return on investment offered. This paper describes this problem and those changes made before and after the "end of the debate about audience measurement" in order to add value to digital advertising spaces. Particularly, the case of online newspapers in analysed and discussed due to its high number of readers and the low incomes perceived.
\end{abstract}

Keywords: Metrics, digital advertising, digital newspapers, revenue; programmatic buying.

1 Doctora en Comunicación Audiovisual y Publicidad por la Universidad Rey Juan Carlos y profesora de grado y Máster en la Universidad Internacional de La Rioja. E-mail: lidia. maestro@unir.net 


\section{Introducción}

Uno de los principales problemas que ha encontrado el desarrollo de la publicidad digital se debe a la falta de consenso sobre su medición. La forma de medir la audiencia debe adaptarse a las nuevas maneras de comunicar y de consumir. La comunicación interactiva que permite el entorno digital obliga a redefinir los mecanismos de medición de audiencias necesarios para realizar la planificación publicitaria. La información y conocimiento de la audiencia es determinante porque actúa como moneda de cambio en la comercialización de espacios publicitarios.

Ante la situación económica de las empresas informativas, la rentabilidad del anuncio de prensa resulta determinante. Este es un aspecto que para los diarios no era importante hasta ahora. Con la notoriedad que las cabeceras tenían se conseguía la fidelidad de lectores y anunciantes. La influencia que ostentaban hacía que los anuncios de prensa fueran rentables para todas las partes implicadas. Actualmente, la influencia ha bajado en internet. Los usuarios siguen las cabeceras web, pero, como Novick apunta a Gómez Borrero ${ }^{2}$, «el problema es que no se ha conseguido que tenga la misma influencia lo que se publica en la red que lo que se publica en el papel». La ventaja que sí aportan las cabeceras web radica en la notoriedad de marca, que viene de la mano de los soportes tradicionales. En un entorno con tanta variedad de soportes, el valor de la marca es determinante. El problema está en cómo se pone valor medible a parámetros que eran bienes intangibles. Lamas ${ }^{3}$ expone que las necesidades de medición responden a dos imperativos fundamentalmente:

- Aportar información que permita el desarrollo del marketing de medios: optimizar los resultados empresariales, mejorando el conocimiento de mercado.

- Establecer la moneda de cambio para la comercialización de los espacios publicitarios en los medios.

En el entorno digital la calidad ha superado a la cantidad como valor predominante. La información cualitativa que aporta hoy día el estudio de audiencias resulta fundamental a la hora de planificar campañas crossmedia que permitan reportar al anunciante un mayor nivel de efectividad. Esto es fundamental en el desarrollo de los medios digitales.

El amplio número de posibilidades de medición permitió inicialmente la aparición de numerosas fuentes de información. Sin embargo, los datos que era posible conocer no eran homogéneos y poco tenían que ver con los paneles consensuados en otros medios. Esta falta de unificación generó que la credibilidad y reputación de este tipo de

2 Gómez Borrero, Mª P. (2010). Prensa e internet. ¿Dónde está el negocio?, 214, Madrid, Escuela de Organización Industrial-MediosOn.

3 Lamas, C. (2010). «Los medios interactivos y su publicidad. La medición de las audiencias», Revista Telos: Cuadernos de Comunicación e Innovación, $\mathrm{n}^{\circ} 82$, 95101 , http://telos.fundaciontelefonica.com/DYC/TELOS/NMEROSANTERIORES/ Nmeros80100/DetalleAnteriores_82TELOS_DOSSIERPV1/seccion=1268\&idioma $=\mathrm{es}$ ES\&id $=2010020409130001$ \&activo $=6$.do 
informaciones no terminara de llegar. La principal consecuencia de la falta de unidad en la medición de la audiencia digital frenó las inversiones publicitarias en este medio inicialmente. A la hora de planificar la publicidad resulta determinante que los datos puedan ser contrastados y ofrecidos al anunciante. El planteamiento de unos estándares que puedan ser aceptados y utilizados por todas las partes afectadas resulta determinante para que se desarrolle la publicidad digital. A partir del 2012, el consenso llego de facto pero no de efecto, como las principales cabeceras indican y los datos corroboran.

\section{Objetivos y metodología}

Esta investigación analiza el problema de la consolidación de la inversión publicitaria que sufren los medios digitales. En concreto, los periódicos digitales, como soportes publicitarios, han perdido rentabilidad frente a sus homólogos en papel. Esta realidad, que afecta a la viabilidad económica de las publicaciones, está relacionada con el cambio de valor que ha sufrido el lector digital respecto al tradicional, al configurarse como moneda de cambio en la comercialización de espacios publicitarios.

El trabajo tiene por objeto de estudio los factores que influyen en la relación económica que se establece entre el lector y la empresa periodística desde el punto de vista de la publicidad. Se delimita el estudio a la prensa digital, puesto que es el medio que ha sufrido un mayor impacto durante la convergencia digital a tenor de la rentabilidad de las cabeceras digitales. Se elabora un cuerpo teórico a través del repaso histórico e investigador de los cambios realizados en el mercado de la venta de espacios publicitarios digitales.

El marco de actuación se centra en el debate de la medición de audiencias digitales, que suponía uno de los principales escollos que impedía que la inversión publicitaria digital se consolidara. Este estudio sirve para evaluar los cambios que han afectado al equilibrio económico de los medios, aunque sus conclusiones afectan a cualquier espacio digital cuyo modelo de negocio se base en la publicidad.

\section{Desarrollo de los espacios publicitarios en la prensa digital}

La migración digital hacia los portales web por parte de los medios de prensa, radio o televisión ha cambiado la organización informativa y la gestión de los diferentes grupos mediáticos. El fenómeno de la convergencia genera cuatro dimensiones implícitas al proceso: dimensión profesional, dimensión comunicativa, dimensión tecnológica y dimensión empresarial. Desde está última óptica, se puede observar una doble vertiente: cómo se produce la coordinación económica y editorial de los diferentes conglomerados y cómo se articula en torno a internet.

La ingente oferta digital no ha cambiado el hecho de que, pese a la digitalización de la información y la aparición de numerosos agentes nuevos en el sector, la difusión sigue concentrada en los mismos medios. Díaz Nosty ${ }^{4}$, citando los estudios de

4 Diaz Nosty, B. (2011), «La crisis en la industria de la prensa. Vida más allá del papel», Revista Telos: Cuadernos de Comunicación e Innovación, $\mathrm{n}^{\circ} 86$, 52-65, 55, http://telos. fundaciontelefonica.com/url-direct/pdf-generator?tipoContenido=articuloTelos\&idContenido= 2011012509430001\&idioma=es 
contenido realizados por Boczkowski ${ }^{5}$, habla de lo que conocemos como paradoja de la abundancia. La multiplicación de la oferta no impide que los usuarios recurran a las fuentes que ya reconocen y valoran. En este sentido, Napoli ${ }^{6}$ nos recuerda la importancia que han adquirido los estudios sobre audiencias y contenido, cada vez más necesarios en el mercado. La necesidad de aumentar o de mejorar cualitativamente las opciones de consumo induce a conocer los gustos, intereses o motivaciones de los usuarios, tanto reales como potenciales. La credibilidad y calidad que los medios tradicionales aportan al usuario hace que sean los mismos actores los que gocen de liderato en el entorno digital; sin embargo, la falta de ingresos hace que esta necesidad de conocimiento sobre los lectores sea imprescindible. La estrategia de planificación de medios se está reorientando al público. En lugar de realizar una selección de medios concreta en los medios online, se ha vuelto dinámica y, como afirma $\mathrm{Papi}^{7}$, «las nuevas tecnologías permiten, sin embargo, insertar publicidad en aquel soporte que está siendo consumido por el usuario que cumple con las características que interesan a la marca (y de las que se dispone información)».

En el sector editorial, la concentración empresarial se ha dispuesto en torno a los medios que acaparan mayor difusión. Los diarios más leídos en España pertenecen a diferentes grupos multimedia, que a su vez reúnen a la mayor parte de la inversión publicitaria. Esta concentración suele producir un exceso y saturación publicitaria en el medio, lo que provoca el rechazo de los usuarios y la reducción de eficacia de las campañas. Esta realidad puede tener efectos contrarios y negativos para el medio. En palabras de Maestro ${ }^{8}$ :

La publicidad seguirá siendo una de las fuentes principales de ingresos y sustento de los diferentes medios, pero debemos prestar especial atención a su gestión ya que un erróneo planteamiento afecta gravemente, entre otros, al producto informativo, a la efectividad del mensaje publicitario o a la notoriedad de la marca, que es el principal reclamo del medio.

El problema de aglutinar la inversión publicitaria en unos medios, que a su vez concentran la propiedad, tiene efectos en los contenidos y en la función publicitaria, pero no conlleva que estos ingresos obtengan una cantidad consolidada dentro de sus balances de cuentas.

5 Boczkowski, P.J. (2010), Imitation in an age of information abundance, Chicago, University of Chicago Press.

6 Napoli, P. (2011), Audience economics. Media institutions and the audience marketplaces, Nueva York, Columbia University Press.

7 Papi Gálvez, N. P. (2014), «Los medios online y la ¿ crisis? de la planificación de medios publicitarios» adComunica, $\mathrm{n}^{\mathrm{a}} 7,29-48,42$, http://www.adcomunicarevista.com/ojs/index.php/ adcomunica/article/view/166

8 MAESTRO, L. (2012). «La saturación publicitaria consecuencia del grado de concentración de los grupos multimedia españoles». En Actas IV Congreso Internacional Latina de Comunicación Social: Comunicación, control y resistencias, 108, Sociedad Latina de Comunicación Social, http://www.revistalatinacs.org/12SLCS/2012_actas/108_Maestro.pdf 
El principal reto al que se han enfrentado los medios desde la aparición de internet, ha sido buscar nuevos modelos de negocio que ayuden a rentabilizar los contenidos. Esta adaptación tiene que darse ante un lector que ha dejado atrás el concepto tradicional que de él se tenía en papel. Se ha convertido en un consumidor de información, un usuario que accede a recursos informativos gratuitos o de pago. La digitalización, hipertextualidad, reticularidad, multimedialidad e interactividad según Scolari ${ }^{9}$, que encontramos en la prensa digital, habilitan al lector para poder asumir nuevas funciones que lo transforman en usuario. A este nuevo enfoque se le une el hecho de que, según Maestro, García Santamaría y Fernández-Beamont ${ }^{10}$, los «modelos comerciales que han desarrollado los grandes agentes digitales están enfocados hacia el cliente final».

Desde la aparición de internet, los diferentes medios se han acercado, con recelo en muchas ocasiones, al mundo digital sin conseguir ningún modelo que resulte rentable a día de hoy o, por lo menos, que tenga la rentabilidad que han perdido los medios tradicionales.

Este estudio se centra en el mundo de la prensa generalista por ser el medio tradicional más consultado por los internautas, lejos de los diferentes portales que las cadenas de televisión y emisoras de radio mantienen en la red. La digitalización ha cambiado la cadena de valor de la información, suponiendo un fuerte impacto en la rentabilidad de las empresas periodísticas. La prensa se enfrenta al mayor reto de su historia, encerrando una enorme paradoja: mientras que asistimos a su mejor momento en cuanto a número de lectores, gracias a la distribución off-online que posibilita un mayor acercamiento al público, la pérdida de valor y de rentabilidad está colocando a cabeceras de todo el mundo al borde del cierre. En el momento en el que tienen más lectores que nunca gracias a la web, también tienen menos facturación, siendo incapaces de monetizar la audiencia y hacer rentables determinadas ediciones. En el Digital Media Latan de 2015 celebrado en México DF, subraya De Llano ${ }^{11}$ cómo Tomas Brunegard, presidente de la Asociación Mundial de Periódicos y Editores de noticias (WAN-IFRA), afirma que en cifras brutas, comparando 2013 y 2014, la circulación de medios en papel aumenta $0,4 \%$ y en medios digitales un $45 \%$. Sin embargo, confirma que los ingresos en papel suponen el $93 \%$ del total.

La importancia que ha adquirido internet como canal informativo, hace necesario que las empresas obtengan un mayor conocimiento de su mercado. Según Soengas,

9 Scolari, C. (2008): «Hipermediaciones. Elementos para una Teoría de la Comunicación Digital Interactiva», Mediaciones Sociales, (8), 167-170.

10 Maestro, L., Santamaría, J. V. y Fernández-Beaumont, J. (2014), «Los retos de las nuevas formas de explotación publicitaria en la financiación de la prensa digital», adComunica, no 7 , 171-189, 108, http://www.adcomunicarevista.com/ojs/index.php/adcomunica/article/view/180

11 De Llano, P (2015) «La industria de los medios digitales busca su modelo de desarrollo», Elpais.com, 24 de Octubre de 2015, México, http://internacional.elpais.com/ internacional/2015/10/23/mexico/1445625932_228140.html 
Vivar y Abuín ${ }^{12}$ «los cambios han transformado el escenario comercial, introduciendo otros códigos y otras formas de relación de los usuarios con los productos y también entre sí»». Esta preocupación ha despertado el interés del ámbito académico. El papel que desarrollan los medios en internet ha sido objeto de estudio de numerosas publicaciones que han formado el cuerpo teórico de una línea de investigación dentro del campo de la comunicación. Toda investigación científica pasa por diferentes estadios, dependiendo de la madurez del fenómeno. Dentro de esas fases, se inscriben las diversas líneas de investigación que afectan al objeto de estudio y que se van planteando según se observan las necesidades. La investigación académica realizada sobre los medios se ha centrado en aspectos que van, desde el nuevo modelo comunicativo o los efectos producidos por los cambios de consumo, hasta el problema de la financiación y los modelos de negocio, que es donde se enmarca este trabajo.

Desde que apareciera la Red, han sido diversas las posturas que las editoriales han tomado. Los modelos que han adoptado los diferentes diarios en su acercamiento al mundo digital han sido variados y cambiantes en el tiempo. Expertos de toda índole han hablado del peligro que corren los medios tradicionales, vaticinando incluso la desaparición de la prensa impresa. Esta tendencia por predecir el final se ha producido cada vez que ha aparecido un nuevo medio. De momento, para cada medio siempre se ha conseguido encontrar un público determinado.

Internet ha convertido a los medios en cibermedios y ha hecho que los periodistas se adapten a los cambios tecnológicos que les exigía el ciberperiodismo. Este canal informativo ha vivido un crecimiento mucho más rápido que cualquier otro medio. En muchos casos, esos cambios que se han visto obligados a realizar, se han llevado a cabo sin los recursos necesarios. La prensa digital ha integrado los géneros tradicionales de la prensa escrita con otros propios del resto de medios de comunicación y particulares de internet. Esto les ha llevado a convertirse en redacciones multimedia. Los nuevos formatos exigen hipertexto, multimedialidad e interactividad: cambios rápidos que se han hecho sin apenas poder reflexionar sobre ellos y sobre los posibles efectos que plantean en el tiempo.

\section{La capacidad de información medida}

A pesar de la falta o no de credibilidad de los contenidos digitales, la mejora comercial dependerá en gran parte de la capacidad de medición de información y, sobre todo, de la gestión de los mismos. En el inicio de internet se auguraba la posibilidad de poder medir todo tipo de informaciones sobre los usuarios. La gran cantidad de datos que buscaban aumentar el volumen de negocio generó un bloqueo del sistema publicitario que impidió el crecimiento de la inversión.

12 Soengas, X., Vivar, H. y Abuín, N. (2015), «Nuevas estrategias de publicidad y marketing para una sociedad hiperconectada», Telos: Revista de Pensamiento sobre Comunicación, Tecnología y Sociedad, $\mathrm{n}^{\circ}$ 101, 115-124, 117, http://telos.fundaciontelefonica.com/DYC/TELOS/ LTIMONMERO/DetalleArtculo_101TELOS_ANALISIS2/seccion=1288\&idioma $=\mathrm{es}$ ES\&id $=2015061812320002$ \&activo $=6$.do 
EnEspaña, Infoadex se encarga de efectuar el control y mediar la actividad publicitaria. Desde 1994 realizan el Estudio de la Inversión Publicitaria en España partiendo de la información que aportan las empresas inversoras y los medios receptores. A partir del año 2000 se elaboran los estudios de agencias de medios y agencias de publicidad en España, que incluyen la inversión real estimada que gestionan las empresas controladas por Infoadex. Analizando la evolución de la inversión publicitaria se ve cómo se han llevado a cabo transacciones de la inversión hacia medios digitales.

Ante la incertidumbre digital, se creó en 1996 la Interactive Advertising Bureau en EEUU con el fin de representar a las empresas del sector publicitario en internet. En 1998 llega a Europa y en 2001 a España. Con el nacimiento de IAB Spain, se establece un organismo dedicado a realizar estudios sobre audiencias digitales. Entre los objetivos de la organización destacan los vinculados con la problemática que padece el sector:

- Fomento de la regulación y estandarización de prácticas.

- Perseguir el crecimiento de la inversión en publicidad y marketing.

- Impulsar la actividad de las empresas asociadas, el desarrollo del marketing y la publicidad interactiva.

La búsqueda de estándares de medición interactiva que permitan comparar diferentes soportes es uno de sus principales anhelos. Para ello, la IAB-USA publicó en 1998 los primeros estándares basados en el mercado americano. En el mercado español hay que destacar el Libro Blanco IAB-AIMC de medición de audiencias digitales realizado conjuntamente por la IAB-Spain y AIMC y en el que se marcan los principios a seguir en la medición de audiencias interactivas.

Actualmente la IAB-Spain tiene dieciséis comisiones que organizan sus líneas de trabajo y que proporcionan diferentes estudios sobre la inversión. En el área digital, los informes que aquí se realizan se han convertido en el mayor referente a la hora de analizar el sector debido a la cantidad y calidad de sus asociados.

En la Asociación para la Investigación de Medios de Comunicación (AIMC) están presentes todos los medios de comunicación. Por ello, desde este organismo se realizan diferentes investigaciones que intentan abordar las necesidades de los diferentes medios. Desde 1996 la AIMC empezó a dar datos sobre la audiencia en internet, que se completaron un año después con encuestas a los internautas. El Estudio General de Medios (EGM) es su mayor aportación investigadora. Contempla datos de prensa, televisión, radio e internet en tres oleadas al año que facilitan la labor de planificación de los diferentes soportes. El problema que para nuestra investigación encontramos en estos datos es que la cabecera digital de El Mundo no se incluye en el estudio. Este hecho dificulta la comparativa de este dato entre las cabeceras y no refleja la realidad total del mercado. 
La Asociación Española de Analítica Web (AEAW) se puso en marcha para promocionar las ventajas de la analítica web dentro del tejido empresarial español. Los profesionales que la integran buscan aportar una unidad metodológica y de herramientas a una disciplina que está en constante evolución.

La OJDinteractiva es la antigua OJD que se encarga de certificar las audiencias de los diferentes medios en internet. Los anunciantes necesitan un control independiente por parte de un auditor que certifique los datos y garantice su inversión. En cuanto a la preocupación que mantiene en la medición, en sus Normas Técnicas de Control define los principios que afectan al control de las mediciones bajo las directrices apuntadas por la IFABC.

La Federación de Comercio Electrónico y Marketing Directo (FECEMD) busca desde finales de los años setenta la promoción y el desarrollo de las diferentes especialidades del comercio electrónico, el marketing y la publicidad directa. El desarrollo tecnológico ha impulsado la evolución de estos campos especialmente.

Internacionalmente destacan otras organizaciones cuyos objetivos se centran en el estudio de audiencias interactivas. La Web Analytics Association (WAA) busca promover la analítica web en todos los campos utilizando la educación y tecnología. Las diferentes acciones que emprenden buscan dotar a este campo de un cuerpo teórico, con metodologías unificadas, amparado en certificaciones y estándares de calidad.

La Internacional Federation of Audit Bureaux of Circulation (IFABC) es un organismo de certificación internacional que está presente en cada país. Su objetivo es controlar las publicaciones de un mercado e intercambiar experiencias en el campo de las mediciones, que permitan desarrollar la publicidad. La IFABC publico sus Web Measurement Standard para controlar las mediciones de audiencias digitales.

Una preocupación común en el tema de las mediciones es el control externo que sirve como garante a los anunciantes. En este sentido, el organismo auditor Media Ratings Council (MRC), que desde la década de los sesenta se erigía como mecanismo de control de las audiencias de radio y televisión del mercado americano, pasó a realizar las auditorias en internet. En este medio, su labor se ha centrado en el control de los adserver, y las empresas que certifican sus datos con sus controles acreditan su calidad. Empresas de medición de audiencia presentes en diferentes países buscan la certificación MRC que valide los datos que aportan ante su mercado.

Como se ve, son numeras las entidades y mecanismos de control que se imponen en los diferentes mercados y que persiguen fines parecidos. Paralelamente a la evolución de las mediciones, el momento del cambio en el mercado publicitario español se produce a partir de 2008. En cuanto a los medios convencionales, la publicidad impresa empieza a bajar y la interactiva comienza a ascender posicionándose en tercer lugar por volumen de inversión a lo largo del primer semestre de 2009. Pero estas 
inversiones sólo llegan a los grandes diarios españoles, que son los que más consiguen atraer la inversión publicitaria. Según Varela ${ }^{13}$, «la proporción de publicidad-difusión en España está equilibrada. Sólo en los grandes diarios españoles la publicidad supera significativamente a la venta al número».

Pero para que la evolución continúe y el crecimiento sea constante, tiene que solucionarse el problema de la medición. La variedad de información inicial llevó a la coexistencia de tres paneles: MediaMetrix, NetRating y NetValue. Ante la dificultad de unificar criterios, se establece de la mano de AIMC e IAB Spain una Comisión de Medición de Audiencias en 2007. El trabajo realizado seguía las pautas implantadas por una iniciativa anterior denominada CASIE (Coalition for Advertising Supported Information and Entertaiment). Durante la década de los noventa, Procter and Gamble puso en marcha esta iniciativa con el fin de sentar las bases de la nueva realidad publicitaria. En 1998 la industria de la publicidad celebró una cumbre en Cincinnati, donde Pete Blackshaw, director digital de $\mathrm{P} \& \mathrm{G}$, mantenía que «uno de los retos de la publicidad interactiva, dado que los consumidores están en un modo muy activo, es que tenemos que preguntarnos si la impresión es la mejor métrica». También auguraba Blackshaw que «la inversión seguirá a la oportunidad». Sin embargo, la realidad demostró que la velocidad de llegada de las inversiones no fue proporcional a la temporalidad con la que se produjo el cambio en los consumos.

La preocupación del sector era unificar una métrica híbrida que combinara los métodos tradicionales censales, las encuestas y los auditores externos. Una combinación site centric y user centric que permita a los medios, agencia, centrales, etc. realizar una comparativa de audiencias, consumos y mercados. La audiencia sigue siendo la moneda de cambio de los medios y es necesario poner en valor los nuevos consumos y usuarios. En la cumbre citada anteriormente se hablaba de las audiencias activas, de cómo su manera de relacionarse había variado el valor que hasta entonces ostentaban. Se ponen en evidencia las incertidumbres de los anunciantes y agencias ante la utilización de los medios digitales. O'Guinn, Allen, Semenik ${ }^{14}$, apuntan como algunos de los problemas tecnológicos la incompatibilidad de estándares técnicos, el ancho de banda ilimitado y una medición que decepciona a todas las partes.

Los anunciantes reclamaban conocer las reacciones de los interlocutores que veían los mensajes, conocer el significado que tenían y el valor que aportan a la marca. Reclamaban más conocimiento sobre su inversión y el retorno de la misma. Según afirman O'Guinn, Allen y Semenik ${ }^{15}$ :

13 Varela, J. (2006), «El fin de la prensa», Cuadernos de periodistas, no8, 17-50, 25, http:// europa.sim.ucm.es/compludoc/AA? articuloId=757114

14 O'GUINN, T., ALLEN, C. y SEMENIK, R. (2007). Publicidad y comunicación integral de marca, México, International Thomson Learning, 107.

15 O Guinn, T., Allen, C. y Semenik, R. (2007). Publicidad y comunicación integral de marca, México, International Thomson Learning, 108. 
Pero esta historia demostró una vez más que la medición del rendimiento por dólar (el rendimiento sobre la inversión, o RSI) en la publicidad (ya sea por internet o no) todavía es escurridizo. Aun cuando mejor que la televisión, internet es fundamentalmente incapaz de ajustarse a las mediciones precisas del rendimiento sobre la inversión en publicidad. Abundan demasiadas variables, demasiado ruido en el sistema, demasiados efectos retardados y demasiadas incertidumbres acerca de quién está realmente allí... siempre resulta difícil medir el impacto de la publicidad. Incluso con toda esta tecnología, todavía es difícil. Y todavía es más difícil decir que tropiezo de ventas proviene de donde.

La situación de la medición en España era compleja. Inicialmente la cuantificación de internautas se realizó por parte de la AIMC-EGM y la medición de server-centric fue llevada a cabo por OJD Electrónica. El mercado disponía de la coexistencia de tres paneles (Media Metrix, NetRating y NetValue). Durante un tiempo el liderazgo lo ostentaba Nielsen que, mediante un acuerdo con la AIMC, utilizaba los datos que manejaba el EGM. Nielsen Online, con su panel NetView y sus marcadores SiteCensus, lideraban el mercado español, pero se complementaban con otro tipo de mediciones como Google Analytics, Omniture o Weborama. Conviene mencionar, por la importancia en el sector de la prensa del mercado local a Netsuus como principal indicador de medición local, de tipo network-centric.

En 2008 se creó un Joint Industry Comité (JIC) en España de la mano de IAB y la AIMC para intentar solucionar los problemas de medición. La llamada Comisión de Medición de Audiencias puesta en marcha publicó el primer Libro blanco de medición de audiencias digitales que sienta como base la figura del individuo como centro de la medición. Posteriormente se crea en 2010 la Mesa de Contratación Digital que propone un concurso para adjudicar un sistema de medición consensuado para el sector. El proyecto ganador, según las bases del concurso, debía:

- Ofrecer un universo de medición acordado por el mercado utilizando las referencias, a partir del Estudio General de Medios.

- Proponer una metodología híbrida de mediciones censales y panelistas que cubra las necesidades del sector y establezca un sistema de medición digital.

- Poner en marcha un comité técnico de clientes que interviniera en las decisiones sobre el mercado local.

- Realizar auditorías externas independientes a las partes.

Para que el mercado publicitario interactivo pudiera incorporarse a la planificación de medios tradicionales se necesitaba establecer unos baremos comunes que pudieran garantizar la inversión del anunciante.

Las empresas que competían eran principalmente Kantar Media, Nielsen Online y ComScore. Cada uno de los sistemas ofrecía una ventaja que los otros no tenía. El resultado de este concurso determinó a ComScore como la empresa de referencia para todos los actores interesados en el proceso. 
A partir del 1 de enero de 2012 y por un periodo de tres años, prorrogable en dos más, ComScore es el operador recomendado. La forma que tiene de operar esta compañía utiliza un panel de internautas y mediciones híbridas. Estas últimas aportan claridad por el uso de una fórmula común que es revisada mensualmente y tiene en cuenta las cookies. El problema de las cookies y su formulación legal en España merece una mención aparte por la influencia que tiene en el desarrollo del mercado publicitario.

El universo que maneja el panel utilizado se ha adaptado al utilizado por el EGM para cumplir así un requisito fundamental del concurso. Pero la principal ventaja de esta empresa recae en la posibilidad de realizar un sistema de seguimiento de la presencia publicitaria sobre el panel que permite conocer la audiencia según los distintos formatos publicitarios. Finalmente, todos los datos aportados serán auditados de forma externa. La AIMC realizará la revisión de la medición de panel (user centric) y la OJD certificará la medición etiquetada (site centric). Posteriormente, en agosto de 2012, ComScore consigue la acreditación del Media Rating Council (MRC) que le aporta la validated campaign essentials (VCE) y certifica la alta calidad de sus datos. Este fue un paso muy importante porque, un año más tarde, las mediciones de su directo competidor, Nielsen, siguen en estudio.

En el mercado europeo cada país ha ido adoptando un consenso diferente sobre las mediciones de audiencias digitales. En casi todos los países se han llevado acuerdos bajo la filosofía JIC que han generado el consenso del sector. Algunos países como Alemania, Bélgica, Holanda y Portugal desarrollaron herramientas propias, mientras que en otros como Francia, Suiza, Italia e Inglaterra, fue Nielsen quien se alzó con la victoria llegando a diferentes acuerdos nacionales. En Francia se unió a Médiametrie y en Suiza con Net Metrix. Los concursos puestos en marcha en Italia los ganó Audiweb, y el convocado por UK Online Measurement Company en Inglaterra tuvo a Nielsen como ganador. En cuanto a herramientas nacionales, en Alemania AGOF implantó un sistema híbrido de información site-centric y encuestas a internautas. Bélgica, a través del JIC denominado Centr d'Information sur le Medias (CIM), estableció los sistemas MetriWeb y MetriProfil con mediciones site centric. En Portugal se impuso el NetPanel de Market Test.

\section{Cambios en la compra de espacios publicitarios}

Pese a todo, no hay que olvidar que los niveles de inversión en publicidad interactiva suponen una cifra mayor de la que manejan las entidades de control y medición. Las cifras cuantitativas reales están lejos de conocerse. La mayor cuota de inversión publicitaria en la web se dirige ya a los buscadores. Google, principal receptor de inversión en buscadores (search), no ofrece datos sobre sus ingresos y además existe otra parte del mercado que está muy atomizado, lo que hace imposible su contabilidad. Esta parte se compone de micro sites con pocos ingresos que, si bien no es probable que lleguen a cumplir la teoría del long tail, sí suponen una cifra importante. La inversión publicitaria continua su ascenso año tras año y las nuevas formas de venta de espacios publicitarios digitales se redireccionan hacia la figura del usuario. 


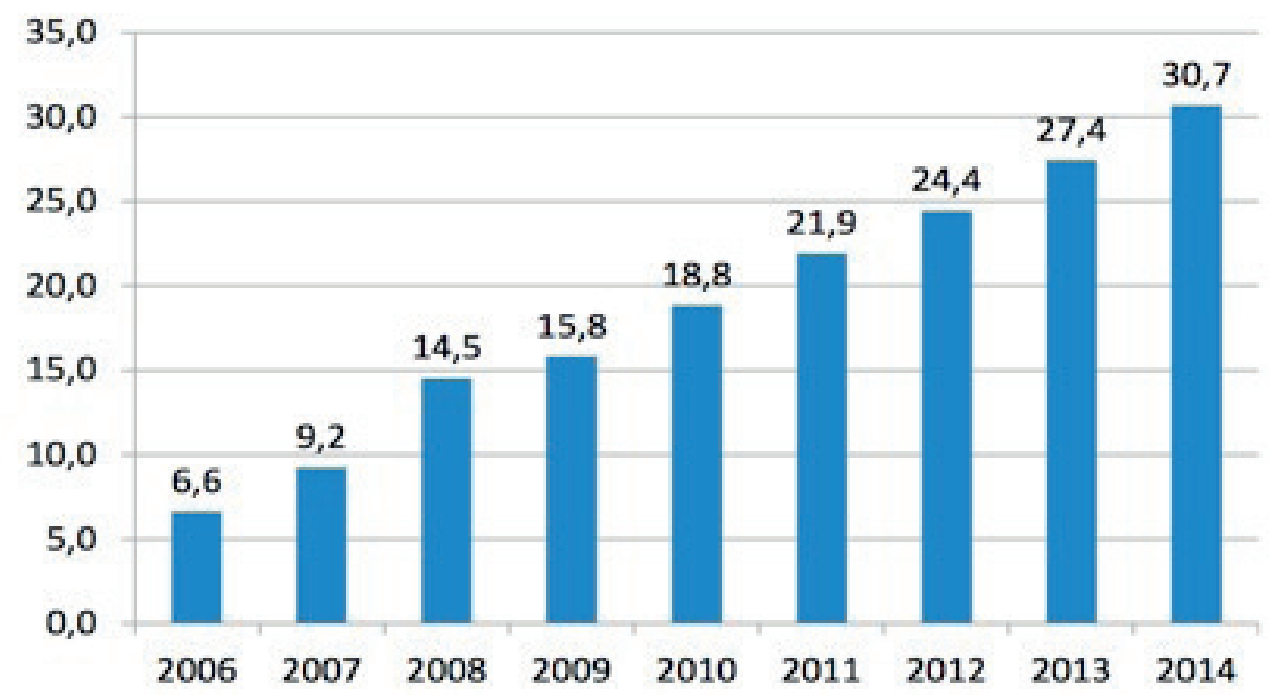

Gráfico 1. Inversión publicitaria en Europa 2014 (Billones de dólares) Fuente: Estudio Inversión publicitaria digital en 2014 IAB Spain ${ }^{16}$

Si se observan los datos de la inversión publicitaria digital por medios, se comprueba que el volumen total para el mercado Europeo de la inversión captada en medios digitales está alcanzando el volumen de la televisión.

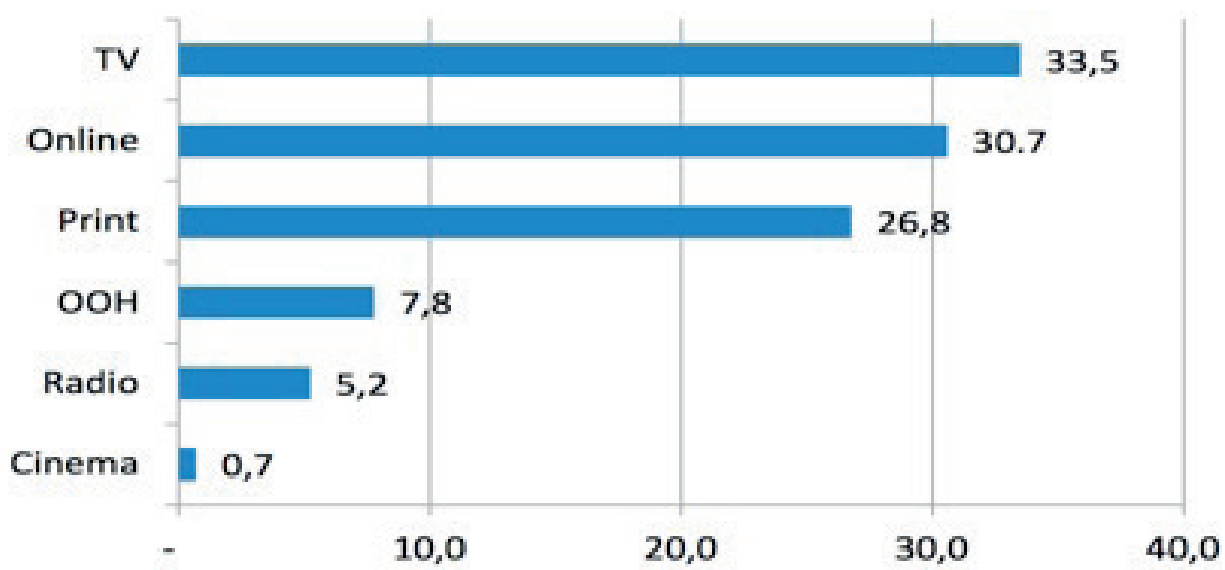

Gráfico 2. Inversión publicitaria digital por medios en Europa 2014 Fuente: IAB Spain Estudio Inversión publicitaria digital en 2014

\footnotetext{
16 Iab Spain (2015), Estudio sobre la Inversión en Publicidad Digital 2014, http://www. iabspain.net/wp-content/uploads/downloads/2015/05/Estudio-Inversion-2015-reducida.pdf
} 
La contratación publicitaria logarítmica y Real Time Bidding (RTB $)^{17}$ sigue en aumento y como afirma Chechu Lasheras, presidente de IAB Spain y subdirector comercial de PRISA Brand Solutions, en las I Jornadas para Editores sobre Compra Programática, «la compra programática no es una opción, es una obligación para los medios y su monetización». Los datos del mercado así lo corroboran. Actualmente en España, el estudio Zenith Vigia ${ }^{18}$ de mayo de 2015 habla de una cifra del 24,7\% de la inversión digital realizada a través de compra programática frente al 15,7\% de volumen de inversión negociada, como afirmaba IAB Spain en su Estudio de Inversión en Publicidad Digital 2014. El estudio IDC "Real-Time Bidding in the United States and Worldwide, 2010-2017" indica que en Estados Unidos un 22,8\% de la venta de publicidad display se ha realizado a través de RTB en 2014, y las cifras previstas para 2015 son del 27,8\% y del 41,3\% en 2017. En Europa, Reino Unido se sitúa a la cabeza con el $20,2 \%$ previsto para 2015 y el $25,1 \%$ para el 2016 .

Aún no están los mercados de compra programática lo suficientemente maduros como para que puedan considerarse un mercado de subastas similar a otros. Las empresas especializadas ofrecen un mix de modelos de compra programática que combina la compra programática directa, con modelos de Real Time Bidding (RTB) o Real Time Audience (RTA). La compra programática esta cambiando la compra de espacios publicitarios y los grupos editoriales se están sumando a esta evolución creando sus propias plataformas para comercializar sus propios espacios publicitarios. Aunque una vez más, aparecen ciertas reservas sobre cómo poder gestionar el inventario más Premium, cómo maximizar el valor de sus lectores o cómo proteger los precios en un entorno con una demanda infinita.

El conocimiento de la audiencia, que según afirma Castelló ${ }^{19}$ que "proporciona información del consumo de medios y soportes así como permite la cuantificación económica del espacio publicitario", adquiere ahora mayor relevancia con la necesidad de reflexionar qué perfiles son más eficientes a la hora de la venta, cuáles mejoran la monetización del medio y cuáles aportan más beneficios a la marca.

17 Real Time Bidding (RTB) es un sistema de pujas online a tiempo real en el que se paga por impresiones conseguidas. Se trata de llegar a los usuarios que están consumiendo un medio en ese momento. El valor de las impresiones varía en función de la cantidad de anunciantes que estén pujando. Todo funciona de manera automática a través del uso de plataformas Ad Exchange donde se introduce la publicidad y los parámetros de público objetivo deseado.

18 Medinaveitia, E. (2015), «ZenithVigía: Mejoran las previsiones de la inversión publicitaria para todos los medios», Bloggin Zenith, http://blogginzenith.zenithmedia.es/zenithvigiamejoran-las-previsiones-de-inversion-publicitaria-para-todos-los-medios/

19 Castelló, A. (2011), « ¿Cuánto vale un fan? El reto de la medición de la audiencia en los social media», Pensar en la publicidad. Revista Internacional de Investigaciones Publicitarias, 4 (2), 89-110, 99, http://revistas.ucm.es/index.php/PEPU/article/view/15887 


\section{Conclusión}

La búsqueda de un modelo de negocio que consiga rentabilizar las ediciones digitales puede tener su respuesta en el valor de los datos. El data que produce todos estos cambios puede suponer el valor diferencial de las prensa digital. La gestión comercial se enfrenta a barreras tecnológicas disruptivas que permitirán monetizar de forma óptima todo el inventario con soluciones adecuadas a la web móvil, In-app y X-device.

El debate sobre la medición digital perdura pese al consenso del sector realizado en 2012. Aunque el malestar por los sistemas de medición que se están realizando está presente aún, las empresas de comunicación han comprobado cómo la unificación en las mediciones no ha conseguido el esperado cambio económico en el valor de la publicidad digital. Debido a la hipervelocidad con la que cambian los tiempos, el consenso alcanzado en la medición aún no aporta una unificación de indicadores que permitan incorporar la información de los canales digitales y de los medios sociales.

Las estrategias basadas en la diversificación de contenidos destinados a diferentes plataformas y audiencias vienen acompañadas de la preocupación por el crecimiento de estas últimas. Mientras se produce el cambio de valor de los espacios digitales, el sector editorial afianza sus lectores, realiza alianzas globales y establece sus propios marketplaces con el fin de intentar sacar todo el provecho económico a los inventarios.

La consolidación de la inversión publicitaria digital no tiene una única respuesta en la unificación de mediciones digitales, como aseguran los editores en base a los datos obtenidos de inversión publicitaria captada por los medios digitales desde 2012. El entorno tecnológico cambiante y repleto de información cualitativa de una audiencia fragmentada que se puede cuantificar y a la que podemos llegar de manera eficaz, parece ser una respuesta para conseguir la monetización de los medios digitales. Las diferentes iniciativas de compra automatizada de espacios publicitarios digitales que se basan en la utilización de los datos están logrando atraer mayores ingresos. La segmentación realizada a través de algoritmos, que tiene en cuenta el perfil y la navegación de los usuarios, ha conseguido simplificar el sistema y acceder a más medios digitales. Todos estos sistemas avanzan en pro de la medición de audiencia y de la consolidación de la inversión publicitaria.

\section{Bibliografía}

Boczkowski, P.J. (2010), Imitation in an age of information abundance, Chicago, University of Chicago Press.

Castelló, A. (2011), « ¿Cuánto vale un fan? El reto de la medición de la audiencia en los social media», Pensar en la publicidad. Revista Internacional de Investigaciones Publicitarias, 4 (2), 89-110, 99, http://revistas.ucm.es/index.php/PEPU/article/ view/15887

De Llano, P (2015) «La industria de los medios digitales busca su modelo de desarrollo», Elpais.com, 24 de Octubre de 2015, México, http://internacional.elpais. com/internacional/2015/10/23/mexico/1445625932_228140.html 
Diaz Nosty, B. (2011), «La crisis en la industria de la prensa. Vida más allá del papel», Revista Telos: Cuadernos de Comunicación e Innovación, no86, 52-65, 55, http:// telos.fundaciontelefonica.com/url-direct/pdf-generator?tipoContenido=articuloTel os\&idContenido $=2011012509430001 \&$ idioma $=$ es

Gómez Borrero, Mª P. (2010:214). Prensa e internet. ¿Dónde está el negocio?, Madrid, Escuela de Organización Industrial-MediosOn, 114.

Iab Spain (2015), Estudio sobre la Inversión en Publicidad Digital 2015, http://www. iabspain.net/wp-content/uploads/downloads/2015/05/Estudio-Inversion-2015reducida.pdf

LAMAS, C.(2010).«Losmediosinteractivosysupublicidad.Lamedicióndelasaudiencias», Revista Telos: Cuadernos de Comunicación e Innovación, $\mathrm{n}^{\circ} 82$, 95-101, http://telos. fundaciontelefonica.com/DYC/TELOS/NMEROSANTERIORES/Nmeros80100/ DetalleAnteriores_82TELOS_DOSSIERPV1/seccion=1268\&idioma $=$ es ES\&id $=2010020409130001 \&$ activo $=6$.do

Maestro, L., García Santamaría, J. V. y Fernández-Beaumont, J. (2014), «Los retos de las nuevas formas de explotación publicitaria en la financiación de la prensa digital». adComunica, no 7, 171-189, 186, http://www.adcomunicarevista.com/ojs/ index.php/adcomunica/article/view/180

MAESTRO, L. (2012). «La saturación publicitaria consecuencia del grado de concentración de los grupos multimedia españoles». En Actas IV Congreso Internacional Latina de Comunicación Social: Comunicación, control y resistencias, Sociedad Latina de Comunicación Social, 108, http://www.revistalatinacs.org/12SLCS/2012_ actas/108_Maestro.pdf

Medinaveitia, E. (2015), «ZenithVigía: Mejoran las previsiones de la inversión publicitaria para todos los medios», Bloggin Zenith, http://blogginzenith. zenithmedia.es/zenithvigia-mejoran-las-previsiones-de-inversion-publicitaria-paratodos-los-medios/

NApoli, P. (2011), Audience economics. Media institutions and the audience marketplaces, Nueva York, Columbia University Press.

Papi Gálvez, N. P. (2014), «Los medios online y la $i$ crisis? de la planificación de medios publicitarios», adComunica, $\mathrm{n}^{\mathrm{a}} 7,29-48,42$, http://www.adcomunicarevista. com/ojs/index.php/adcomunica/article/view/166

Soengas, X., Vivar, H. yAbuín, N. (2015), «Nuevas estrategias de publicidad y marketing paraunasociedadhiperconectada»,Telos:RevistadePensamientosobreComunicación, Tecnología y Sociedad, $\mathrm{n}^{\circ}$ 101, 115-124, 117, http://telos.fundaciontelefonica. com/DYC/TELOS/LTIMONMERO/DetalleArtculo_101TELOS_ANALISIS2/ seccion $=1288 \&$ idioma $=$ es_ES\&id $=201506181232000 \overline{2} \&$ activo $=6 . \overline{\mathrm{do}}$

Scolari, C. (2008): «Hipermediaciones. Elementos para una Teoría de la Comunicación Digital Interactiva», Mediaciones Sociales, (8), 167-170.

O'Guinn, T., Allen, C. y Semenik, R. (2007). Publicidad y comunicación integral de marca, 108, México, International Thomson Learning.

Varela, J. (2006), «El fin de la prensa», Cuadernos de periodistas, n' 8 , 17-50, 25 http://europa.sim.ucm.es/compludoc/AA?articuloId=757114 